\title{
Promovendo melhorias na Comunidade Democrática Cidadã por meio de avaliação heurística
}

\author{
Victor Félix Arinos, Cristiano Maciel, Jivago Medeiros
}

Instituto de Computação - Universidade Federal de Mato Grosso (UFMT)

Av. Fernando Corrêa da Costa, no 2367 - Bairro Boa Esperança - Cuiabá - MT - Brasil

\{victorfelixar, crismac, jivagomr\}egmail.com

\begin{abstract}
The use of Information and Communication Technologies (ICTs) has increased access to information and user interaction on the internet. This scenario facilitates the growth of virtual communities with different purposes, such as Comunidade Democrática Cidadã (CDC) which seeks to bring the state closer to the citizens through a consultative-deliberative system. Thus, this paper proposes the application of a heuristic evaluation in the CDC platform, analyzing the errors found and their degree of severity, as well as assigning a classification for the problems and proposing a possible solution to it, seeking to increase the adherence of users and decrease the difficulties encountered.
\end{abstract}

Resumo. A utilização de Tecnologias da Informação e Comunicação (TIC's) vem auxiliando o processo de acesso a informação e interação de usuários. Este cenário facilita o crescimento de comunidades virtuais com diversos propósitos, como a Comunidade Democrática Cidadã (CDC) que busca a aproximação da máquina administrativa com os cidadãos através de um sistema consultivodeliberativo. Neste sentido, este trabalho propõe a aplicação de uma Avaliação Heurística na plataforma CDC, analisando os erros encontrados e seu grau de severidade, além de atribuir uma classificação para os problemas e propor uma possível solução, buscando aumentar a adesão dos usuários e diminuir as dificuldades encontradas.

\section{Introdução}

A participação dos cidadãos no processo de dicussão, escolha e tomada de decissão é um fator importante da democracia [Garcia et al. 2005, Maciel et al. 2016], o qual auxilia governo e população a convergir num resultado satisfatório para ambos os lados. Este processo de interação entre população e máquina administrativa possui grandes desafios devido a dificuldade em estabelecer diágolos e propostas num mesmo ambiente [Slaviero et al. 2012]. Uma forma de contornar esta dificuldade está na utilização de comunidades virtuais [Maciel 2008]. Desta forma, podemos viabilizar a discussão em torno de um assunto ou problema através da democracia eletrônica (e-Democracia) nestas comunidades [Hrustek et al. 2015]. Dentre as diversas plataformas de interação governo-cidadão existentes está a Comunidade Democrática Cidadã (CDC), caracterizada por ser uma comunidade virtual para debates e votações. Contudo, a forma como os usuários utilizam e percebem uma determinada interface pode afetar a sua satisfação [Marques et al. 2015]. Neste sentido, este trabalho propõe uma Avaliação Heurística da plataforma CDC, analisando os problemas de usabilidade encontrados. Além disso, os 
erros são classificados quanto ao seu grau de severidade e perfil afetado pelo problema, analisando quais as heurísticas e perfis com maior quantidade de problemas encontrados. Ao final, alguns erros são discutidos, sendo apontados possíveis re-designs como solução, servindo de apoio para a adaptação da plataforma e, consequentemente, melhor adesão dos usuários.

Este trabalho está organizado em 6 seções, sendo a segunda seção dedicada para o Referencial Teórico; a terceira seção voltada para descrever a metodologia; a quarta seção utilzada para apresentação dos resultados; a quinta seção dedicada para discussão e conclusão e, por último, a bibliografia utilizada.

\section{Usabilidade e Avaliação Heurística}

O termo usabilidade pode ser definido como uma medida utilizada para aferir o quanto um sistema de computador possibilita a um usuário, em um dado contexto, alcançar objetivos específicos, gerando concomitantemente sentimentos de felicidade/satisfação [ISO-9241-210 2019]. Para Nielsen (1990), a usabilidade de um sistema e os seus aspectos devem ser analisadas em todos os casos onde há interação humano. Uma forma de avaliar a usabiliadade de uma plataforma se dá através de um método de inspeção denominado Avaliação Heurística. Esta avaliação é caracterizada como uma lista de regras a serem analisadas num determinado objeto de estudo [Nielsen and Molich 1990]. Neste método são utilizadas 10 heurísticas consideradas de caráter genérico, sendo assim adaptáveis para o contexto em que estão sendo aplicadas [Mosqueira-Rey et al. 2018], além de não ter usuários reais como fonte de observação, sendo que avaliadores examinam a interface simulando usuários [Nielsen 1994b]. Além disso, a inspeção por Avaliação Heurística possui como característica o baixo custo, rapidez e facilidade de aplicação [Bim et al. 2016] com grande capacidade de identificar e analisar erros ou problemas [Barbosa and Silva 2010].

Assim, diversos estudos propõem adaptações das heurísticas de Nilsen para contextos específicos, como no caso de sites governamentais. Especificamente em relação a interface de sistemas voltado para interação governo-cidadão, tendo em vista o escopo deste trabalho, tem-se alguns estudos interessantes. Marques et al. (2015), por exemplo, aplicaram métodos distintos de inspeção em diferentes sites governamentais brasileiros, comparando o resultado das técnicas utilizadas, além de elencar os erros encontrados. Dessa forma, diversos modelos de Avaliação Heurística podem ser criados a partir da adaptação das 10 heurísticas de Nielsen de acordo com o objeto de estudo.

\section{Metodologia}

Nesta seção serão elencadas as metodologias utilizadas neste estudo.

\subsection{Comunidade Democrática Cidadã}

O aumento da utilização de Tecnologias da Informação e da Comunicação (TIC's), em especial a internet, auxiliou o processo de criação e acesso a conteúdo [Maciel et al. 2004]. Este cenário facilitou a criação e disseminação de comunidades virtuais, definidas como grupo de pessoas identificáveis e dispersas geograficamente [Maciel et al. 2004, de Oliveira Bueno and Anacleto 2017]. Dentre as comunidades virtuais utilizadas para interação governo-cidadão está a Comunidade Democrática Cidadã, caracterizada por utilizar o Modelo Interativo Governo-Cidadão, o qual representa as distintas fases de um 
processo consultivo e deliberativo [Maciel 2008]. Este processo utiliza como base a discussão argumentativa, onde opiniões são apresentadas através de comentários e, posteriormente, há a possibilidade de votação para escolha entre demandas ou soluções propostas para os problemas [Maciel et al. 2018].

Nesta plataforma os usuários encontram, após cadastro, um espaço para diálogo estruturado por temáticas, tais quais cultura, política, saúde, transportes, entre outros. Neste espaço é possível iniciar debates, como a discussão de demandas, onde há o registro de opiniões do cidadão. Além disso, ao final das deliberações de um debate, temos o processo de votação. Assim, tem-se na plataforma um espaço para exposição de demandas capaz de registrar opiniões e o voto no final do processo, configurando as diversas fases do processo de consulta e deliberação. $\mathrm{O}$ ambiente proposta pela $\mathrm{CDC}$, em especial devido a sua característica de reprodução de ações consultivas-deliberativas, permitiu a utilização da aplicação como ambiente de experimentação na área de e-democracia, sucitando novos trabalhos na área. Martins et al. (2015), por exemplo, propôs a adição de um sistema de recomendação como forma de apoiar a participação dos usuários na Comunidade Democrática Cidadã. Atualmente a Comunidade Democrática Cidadã pode ser acessada através do seguinte endereço: http://cdc.ic.ufmt.br/CDC/ .

\subsection{Avaliação Heurística}

Para este estudo foi aplicado o método de inspeção denominado Avaliação Heurística, o qual busca localizar problemas de usabilidade de uma interface através de um conjunto de regras (heurísticas) pré-defindidas [de Lima Salgado et al. 2016, Nielsen 1994a]. Para isso, foi utilizado um formulário proposto por Maciel et al. (2004), composto por 10 heurísticas:

1. Visibilidade do status do sistema: Avaliar se mantem o usuário informado;

2. Compatibilidade do sistema com o mundo real: Avaliar se a linguagem utilizada é próxima do público alvo;

3. Controle do usuário e liberdade: Avaliar se os usuários possuem a sensação de que controlam o site;

4. Consistência e Padrões: Avaliar se há padronização nos elementos do domínio;

5. Prevenção de erros: Avaliar se os elementos dispostos evitam erros;

6. Reconhecimento ao invés de lembrança: Avaliar se é necessário lembrar de informações exibidas no site;

7. Flexibilidade e eficiência de uso: Avaliar se o sistema é utilizável por todos os perfis de usuários;

8. Estética e design minimalista: Avaliar se o sistema utiliza elementos desnecessários que possam distrair ou confundir o usuário;

9. Ajudar os usuários a reconhecer, diagnosticar e corrigir erros: Avaliar se o sistema exibe mensagens de erro com informações suficientes para o usuário corrigir o problema;

10. Ajuda e documentação: Avaliar se há o recurso de ajuda (help);

Cada seção possui especificações do problema encontrado, como o grau de serevidade (Sem importância; Cosmético; Simples; Grave; Catastrófico), a frequência do problema (Principal; Secundário), o tipo de usuário afetado (Geral; Preliminar; Especial) [Nielsen 1994b]. Além disso, os problemas foram descritos quanto a aspectos como 
contexto, efeitos sobre o usuário, causa e re-design possível para facilitar o mapeamente e classificação [Nielsen 1994b] [Maciel et al. 2004]. Para a realização dos testes foram utilizados 35 avaliadores divergindo entre experiencia com diferentes perfis (alunos de graduação, desenvolvedores, analistas). Todos os avaliadores que aplicaram o método receberam uma explicação sobre este estudo, além de detalhes sobre o processo de inspeção com Avaliação Heurística. Os erros encontrados com a aplicação do método de inspeção, realizado por avaliadores com conhecimento em usabilidade, foram analisados e agrupados. Esta etapa foi realizada pelos autores do artigo, especialistas com atuação na área de usabilidade, buscando evidenciar os erros únicos encontrados.

\section{Resultados}

Nesta seção serão apresentados os resultados da Avaliação Heurística e a proposta do possível re-design de alguns dos erros levantados.

\subsection{Avaliação Heurística}

Diversos erros foram localizados durante a realização da Avaliação Heurística na plataforma CDC. No total foram encontrados 122 erros pelos 35 avaliadores, variando de heurísticas e grau de severidade. Deste total, apenas 71 erros eram únicos, ou seja, não estavam repetidos. Na Tabela 1 temos tabulados a quantidade de erros únicos e o grau de severidade encontrado para cada heurística.

\section{Tabela 1. Número de erros únicos identificados}

Heurísticas/Seção

Visibilidade do status do sistema

Compatibilidade do sistema com o mundo real

Controle do usuário e liberdade

Consistência e Padrões

Prevenção de erros

Reconhecimento ao invés de lembrança

Flexibilidade e eficiência de uso

Estética e design minimalista

Ajudar os usuários a reconhecer, diagnosticar e corrigir erros

Ajuda e documentação

Total

$\begin{array}{cccccc}\text { Grau de Severidade } & \\ \text { Sem importância } & \text { Cosmético } & \text { Simples } & \text { Grave } & \text { Catastrófico } & \text { Total } \\ 0 & 2 & 3 & 4 & 1 & 10 \\ 0 & 2 & 3 & 1 & 0 & 6 \\ 0 & 1 & 3 & 2 & 1 & 7 \\ 1 & 3 & 1 & 3 & 0 & 8 \\ 0 & 0 & 4 & 6 & 1 & 11 \\ 0 & 1 & 6 & 1 & 0 & 8 \\ 0 & 0 & 3 & 0 & 0 & 3 \\ 0 & 1 & 2 & 2 & 0 & 5 \\ 1 & 1 & 3 & 3 & 0 & 8 \\ 0 & 0 & 3 & 2 & 0 & 5 \\ 2 & 11 & 31 & 24 & 3 & 71\end{array}$

Pode-se observar que a heurística com maior quantidade de problemas é a de "Prevenção de erros", com 11 problemas identificados, o que equivale a aproximadamente 11\%. Em seguida, temos a "Visibilidade do status do sistema" com 10 erros (14\%). Todos os outros aspectos avaliados resultaram em menos de 10 problemas. "Estética e design minimalista" resultou em uma das seções com o menor número de erros juntamente com "Ajuda e documentação". Quanto ao grau de severidade, pode-se notar que grande parte dos erros foram classificados como Simples, resultando em 31 problemas dessa classificação (41\%). Em seguida temos o grau de severidade Grave com 24 erros, aparecendo, em especial, na heurística "Prevenção de erros", representando mais da metade dos problemas apresentados dessa seção. Os erros classificados como Sem importância e Catastróficos possuem $2(3 \%)$ e $3(4 \%)$ problemas, respectivamente. Os erros sem importância foram localizados nas heurísticas "Consistência e Padrões" e "Ajudar os usuários a reconhecer, diagnosticar e corrigir erros". Isto se deve devido ao baixo número de erros por falta de padronização da plataforma, que utiliza poucos ícones para ilustração das ações. 


\subsection{Problemas e re-design}

Os problemas encontrados na plataforma CDC foram classificados em sua maioria (46 erros - 64\%) do tipo Problema Geral, ou seja, que poderiam ocorrer com qualquer usuário que utilizasse a plataforma. Em seguida temos a classificação Problema preliminar, caracterizada por afetar somente usuários inexperientes com tecnologia com 23 erros (32\%), estando por último Problema Especial com 2 erros (aproximadamente 3\%). Neste último caso temos que os problemas encontrados estão relacionados a acessibilidade. A plataforma CDC não apresenta opções para usuários com problemas visuais ou outros portadores de necessidades especiais, diminuindo o acesso do público a ferramenta. Esta situação pode se tornar um problema, em especial devido a característica democrática que a plataforma deve oferecer a todos os usuários que desejam interagir e opiniar. Um problema frequentemente elencado foi a utilização de letras pequenas para diversas áreas do sistema, em especial na exibição de mensagens de erro, dificultando o entendimento do usuário caso o mesmo não consiga localizá-las. Um dos erros catastróficos apontado está na tela de visualização de perfil, no qual os dados dos usuários cadastrados podem ser acessados por outras pessoas, podendo levar a coleta de dados sensíveis por exemplo. Uma solução é limitar a exibição para apenas dados não sensíveis ou de domínio público.

\section{Discussão e Conclusão}

Um dos principais fatores para adesão de plataformas é a usabilidade que ela apresenta e seus impactos nos usuários. Esta situação se torna mais crítica quando lidamos com plataformas de cunho democrático, onde os usuários devem se sentir confortáveis em utilizar estas ferramentas para facilitar a adesão e a interação entre cidadão e máquina administrativa. Percebe-se através deste estudo que métodos de avaliação de usabilidade, como a inspeção através da Avaliação Heurística, permitem aos analistas e desenvolvedores de software adaptarem e melhorarem as plataformas. Isto se comprova através da quantidade de erros encontrados na plataforma de interação CDC. Observa-se que grande parte dos erros encontrados podem frustrar o usuário, em especial por causa da heurística mais afetada pelos erros (Prevenção de erros), o qual demonstra que não há meios na plataforma para impedir o usuário de tomar decisões errôneas. Além disso, em grande parte do sistema não é apresentado o status atual das ações, resultando em confusão por parte do usuário. Apesar de parecer irrelevante, estes erros podem afastar os usuários de uma plataforma, diminuindo a utilização da mesma. Este cenário deve ser evitado, em especial num contexto democrático como o da CDC. Cabe reforçar que a CDC é uma aplicação desenvolvida e experimentada no contexto educacional e está disponível para parcerias em projetos.

Dentre os possíveis trabalhos futuros podemos elencar a análise da user experience resultante da utilização da plataforma, assim como os possíveis impactos sobre o usuário, além da utilização de outras técnicas de avaliação e inspeção de usabilidade.

\section{Referências}

Barbosa, S. and Silva, B. (2010). Interação humano-computador. Elsevier Brasil.

Bim, S., Salgado, L., and Leitão, C. (2016). Evaluation by inspection: Comparing methods of practical, cognitive and semiotic basis. In Proceedings of the 15th Brazilian Symposium on Human Factors in Computing Systems, IHC '16. 
de Lima Salgado, A., Rodrigues, S. S., and Fortes, R. P. M. (2016). Evolving heuristic evaluation for multiple contexts and audiences: Perspectives from a mapping study. In Proceedings of the 34th ACM International Conference on the Design of Communication. ACM.

de Oliveira Bueno, A. and Anacleto, J. C. (2017). Municipal virtual communities (muvic): Expanding cities to the virtual world. In Proceedings of the XVI Brazilian Symposium on Human Factors in Computing Systems. ACM.

Garcia, A. C. B., Maciel, C., and Pinto, F. B. (2005). A quality inspection method to evaluate e-government sites. In International Conference on Electronic Government, pages 198-209. Springer.

Hrustek, N. Z., Musa, A., and Mekovec, R. (2015). The perception of e-democracy and e-participation at the individual level: A croatian example. In Proceedings of the 2015 2Nd International Conference on Electronic Governance and Open Society: Challenges in Eurasia. ACM.

ISO-9241-210 (2019). Ergonomics of human-system interaction — part 210: Humancentred design for interactive systems. Technical report.

Maciel, C. (2008). Um método para mensurar o grau de maturidade da tomada de decisão e-democrática. Niterói (RJ), 230p. Thesis (D. Sc. in Computer Science)-Programa de Pós-Graduação em Ciência da Computação, UFF, Niterói-RJ-Brazil (In Portuguese).

Maciel, C., Cappelli, C., Slaviero, C., and Garcia, A. C. B. (2016). Technologies for popular participation: A research agenda. In Proceedings of the 17th International Digital Government Research Conference on Digital Government Research. ACM.

Maciel, C., Nogueira, J. L. T., Ciuffo, L. N., and Garcia, A. C. B. (2004). Avaliação heurística de sítios na web. Escola de Informática do SBC-Centrooeste.

Maciel, C., Roque, L., and Garcia, A. C. B. (2018). Maturity in decision-making: A method to measure e-participation systems in virtual communities. International Journal of Web Based Communities, 14.

Marques, V., Ferreira, B., Pinho, J., Oliveira, R., and Maciel, C. (2015). Inspeção da interação em sítios governamentais: uma comparação entre métodos. In Anais do XI Simpósio Brasileiro de Sistemas de Informação. SBC.

Mosqueira-Rey, E., Alonso-Ríos, D., and Moret-Bonillo, V. (2018). A heuristic evaluation of the user and programming interfaces of a sleep medicine application. In Proceedings of the 33rd Annual ACM Symposium on Applied Computing. ACM.

Nielsen, J. (1994a). Enhancing the explanatory power of usability heuristics. In Proceedings of the SIGCHI conference on Human Factors in Computing Systems. ACM.

Nielsen, J. (1994b). Usability inspection methods. In Conference companion on Human factors in computing systems, pages 413-414. ACM.

Nielsen, J. and Molich, R. (1990). Heuristic evaluation of user interfaces. In Proceedings of the SIGCHI Conference on Human Factors in Computing Systems. ACM.

Slaviero, C., Garcia, A. C. B., and Maciel, C. (2012). Exploiting eparticipation using an ontological approach. In 4th International Conference on Electronic Participation, pages 144-155. Springer. 